Original Article

\title{
IN VITRO ANTILEISHMANIAL ACTIVITIES OF THREE MEDICINAL PLANTS: ARGEMONE MEXICANA, MURRAYA KOENIGII AND CINNAMOMUM TAMALA AGAINST MILTEFOSINE RESISTANT PROMASTIGOTES OF LEISHMANIA DONOVANI PARASITES
}

\author{
SIRIN SALMA SULTANA \\ Departmental of Zoology, Animal Behaviour and Natural Product Research Laboratory, West Bengal State University, Berunanpukuria, \\ Malikapur, Barasat, 24 Parganas (North), Kolkata 700126 \\ Emails: sirin45632@gmail.com \\ Received: 01 Feb 2021, Revised and Accepted: 30 Jul 2021
}

ABSTRACT

Objective: Leishmaniasis is one of the neglected tropical diseases in terms of drug development and discovery. Non-responsiveness and resistance to the drug in Leishmania species need to develop new antileishmanial potentials; herbal medicines could be the alternative one.

Methods: In the present study, semi-purified fractions were prepared from the traditionally used three medicinal plants of India: Argemone mexicana (aerial shoot), Murraya koenigii (stem), and Cinnamomum tamala (bark) by using multiple solvent systems (non-polar to polar, beginning with petroleum ether followed by $n$-hexane, benzene, and chloroform) and an effort was given to assess the leishmanicidal activities against Leishmania donovani miltefosine resistant HePC-R (Ld/MIL-30) promastigotes in vitro and the IC50 concentrations were estimated.

Results: The study revealed that the semi-purified fractions of A. mexicana, M. koenigii, and C. tamala have effective antileishmanial activities and the $50 \%$ inhibitory concentrations (IC50) are $50 \mu \mathrm{g} / \mathrm{ml}, 98 \mu \mathrm{g} / \mathrm{ml}$, and $200 \mu \mathrm{g} / \mathrm{ml}$, respectively. At these (IC50) concentrations, these plant semipurified fractions were found to interfere in lipid and protein biosynthesis, alter cell morphology, DNA content, mitochondrial membrane potential, generating ROS, and apoptosis in promastigotes. The semi-purified fractions were also found noticeably non-toxic towards host splenocytes.

Conclusion: These results could suggest that A. mexicana, M. koenigii, and C. tamala could carry potential novel compounds for the development of new drugs against Leishmaniasis.

Keywords: Plant semi-purified fractions, Leishmania, Miltefosine resistant, Apoptosis, ROS

(c) 2021 The Authors. Published by Innovare Academic Sciences Pvt Ltd. This is an open-access article under the CC BY license (https://creativecommons.org/licenses/by/4.0/) DOI: https://dx.doi.org/10.22159/ijpps.2021v13i9.42349. Journal homepage: https://innovareacademics.in/journals/index.php/ijpps.

\section{INTRODUCTION}

Leishmaniasis is one of the neglected tropical diseases in terms of drug discovery and development and is endemic in our country (India) [1]. The chemotherapy used to treat this disease has been proved to be highly toxic and has persistence of resistance issues. Miltefosine (MIL) or Hexadecylphosphocholine, is the first orally administrable anti-leishmanial drug but due to its long half-life, it is highly susceptible to resistance. Parasites with decreased drug vulnerability have been associated with treatment failure [2-4]. The emergence of drug resistance is the biggest threat to the successful treatment of leishmaniasis. As a consequence, the need for ideal leishmanicidal molecules to overcome resistance issues has notably increased in recent years.

The native medicinal plants and plant-derived drugs are the potential source of alternative medicine and are extensively used for the treatment of various health ailments $[5,6]$. About $25 \%$ of prescribed drugs were obtained from plants with or without further modification [7, 8]. Use of the medicinal plants is a core component at the primary health care level due to cheaper and easier availability, acceptability, compatibility, and affordability and with less or no side effects. A broad range of available plant species was reported with potentially active leishmanicidal activities $[7,9,10]$.

In the present work, an attempt had been made to evaluate the leishmanicidal activities of semi-purified fractions of three different plants Argemone mexicana (Papaveraceae), Murraya koenigii (Rutaceae), and Cinnamomum tamala (Lauraceae), which are also used as traditional medicine in India. Argemone mexicana is an annual herb with an extremely prickly branched stem and showy yellow flowers [11]. In Homeopathy, the medicine prepared from this herb is used for the treatment against tapeworm [11]. Murraya koenigii is a deciduous shrub with a short trunk and smooth, greyish, or brown bark and has a dense shady crown [12]. It is widely used for culinary purposes and as traditional medicine. Also, it has multiple scientific reports of its medicinal properties [13, 14]. Cinnamomum tamala is a medium-sized evergreen tropical tree with small, yellowish flowers [15]. The leaves of $C$. tamala have been used for flavoring food and it has been used in traditional medicines as an astringent, stimulant, diuretic, carminative, and cardiac disorders [15]. This present study aimed to find out the active antileishmanial potentials against miltefosine resistant Leishmania donovani parasites from medicinal plants of India.

\section{MATERIALS AND METHODS}

\section{Collection and identification of plant material}

The plant materials of Argemone mexicana (Papaveraceae) were collected from the village Gobindapur of Malda district in April 2015; Murraya koenigii (Rutaceae) plant was collected from the village Gazole of Malda district in July 2015 and the plant materials of Cinnamomum tamala (Lauraceae) were collected from the village Checkpost of Malda district in September 2015. The samples were identified in the Animal behavior and Natural product research laboratory, West Bengal State University, WB, India, with the plant identification numbers (SSS23413), (SSS3414), (SSS3416), respectively.

\section{Phytochemical work-up procedures}

The dried coarsely ground plant materials were extracted following the protocol for the enrichment of compounds. The isolated aerial part of A. mexicana, the stem of M. koenigii, and the bark of $C$. tamala were dried at a hot air oven $\left(50-55^{\circ} \mathrm{C}\right)$ for $48 \mathrm{~h}$ and crushed with a mixture grinder. The dried powder materials of the three plants were mixed with petroleum ether $(1: 5 \mathrm{~W} / \mathrm{V})$ individually, extracted in Soxhlet apparatus for $2 \mathrm{~d}$ at room temperatures, and then filtered 
separately with Whatman No. 1 filter paper. The filtrated phytochemicals were concentrated from solvent petroleum ether $(1: 5 \mathrm{~W} / \mathrm{V})$ using the rotary evaporator (Buchi, model no. B 100). The leftover materials were dried again at room temperature and were sequentially partitioned into $n$-hexane $(1: 5 \mathrm{~W} / \mathrm{V})$, benzene $(1: 5$ $\mathrm{W} / \mathrm{V}$ ) and finally chloroform $(1: 5 \mathrm{~W} / \mathrm{V}$ ) for $3 \mathrm{~d}$ (each step) $[16,17]$. Each fraction was concentrated to dryness by evaporation with the Buchi rotary evaporator (Model B 100) of the solvent. Activated charcoal was used to remove the unnecessary pigments from chloroform-derived phytochemicals for bioassay-guided activity studies.

\section{Sample preparation}

The semi-purified fractions were dissolved in sterile dimethyl sulfoxide (DMSO). Appropriate stock solutions $(20 \mathrm{mg} / \mathrm{ml})$ were stored at $-20^{\circ} \mathrm{C}$ until use (antileishmanial activity studies).

\section{Cell biology methods}

\section{Parasite maintenance}

Leishmania donovani drug-resistant parasites HePC-R (Ld/MIL-30) strain was used for experimental purposes. L. donovani miltefosineresistant strain (named HePC-R) was developed in our laboratory from the bone marrow aspirates of a relapsed miltefosine-treated visceral leishmaniasis (VL) patient from West Bengal, India, by transforming and maintaining in continuous drug pressure with stepwise increasing doses of miltefosine as per the approval (reference number WBSU-IEC/06, dated 24 April 2012) described earlier [18]. Parasites were maintained [19], in vitro in complete M199 medium, supplemented with $1 \%$ penicillin-streptomycin and $10 \%$ fetal calf serum at requisite temperature $\left(22{ }^{\circ} \mathrm{C}\right)$. For experimental purposes, log-phase promastigotes were sub-cultured every $72-96 \mathrm{~h}$, inoculums being $5 \times 10^{5} / \mathrm{ml}$.

\section{Promastigote viability assay by 7AAD staining in FACS}

Cytotoxic effect was evaluated on promastigotes $\left(2 \times 10^{5}\right.$ cells/well $)$ without (control) or with $50 \mu \mathrm{g} / \mathrm{ml}$ concentration of all three semipurified fractions in vitro, using 7-amino actinomycin D (7-AAD) as described earlier [19] in FACS. Samples were run on a flow cytometer (BD FACSVerse ${ }^{\mathrm{TM}}$, BD Biosciences, USA) and analyzed by Flowing software, version 2.5 [20]. The unstained population was taken as a reference.

Effects of semi-purified fractions of A. mexicana, M. koenigii, and C. tamala on miltefosine resistant $L$. donovani promastigotes (HePC-R) and determination of IC50

The $50 \%$ inhibitory concentration (IC50) on L. donovani HePC-R promastigotes and cytotoxic effect on murine splenocytes without or with increasing concentration of semi-purified fractions of $A$. mexicana, M. koenigii, and C. tamala was estimated by MTT [3-(4,5dimethylthiazol-2-yl)-2,5-diphenyltetrazolium bromide] method with the doses started from $50 \mu \mathrm{g} / \mathrm{ml}$ to $400 \mu \mathrm{g} / \mathrm{ml}$ described earlier $[19,21]$. Three independent experiments were performed in triplicate for each set. The $50 \%$ inhibitory concentrations of semipurified fractions have been determined from the graph of percent inhibition against increasing concentrations. Statistical analyses for all experiments were performed by one-way ANOVA in R-3.4.1 software followed by Tukey's test.

\section{Effect on protein content in promastigotes}

The protein contents of promastigotes treated without (control) or with IC50 concentrations of these three semi-purified fractions were assessed by staining with fluorescein isothiocyanate (FITC), an acidic dye that covalently binds with the positively charged proteins as described earlier [20] in FACS. The acquisition was done in a flow cytometer (BD FACSVerse ${ }^{\mathrm{TM}}, \mathrm{BD}$ Biosciences, CA, USA) and was analyzed by Flowing 2.5 version software19. The unstained population was taken as a reference.

\section{Effect on neutral hydrophobic lipids in promastigotes}

Neutral lipid contents were assessed as described earlier [19]. Neutral lipids were assessed in promastigotes $\left(1 \times 10^{6}\right.$ cells $\left./ \mathrm{ml}\right)$ treated without or with IC50 concentrations of these three semi- purified fractions for $48 \mathrm{~h}$ by staining with Nile red (Sigma) and acquired in a flow cytometer (BD FACSAria ${ }^{\mathrm{TM}}$, BD Biosciences, USA) and analyzed by Flowing 2.5 version software [20].

\section{Analysis of cell cycle phases}

In vitro grown of promastigotes $\left(1 \times 10^{6}\right)$ were incubated without or with IC50 concentrations of semi-purified fractions and were analyzed for cell cycle progression by fluorescent dye propidium iodide [19] that binds to the DNA. The acquisition was performed on a flow cytometer (BD FACSVerse, BD Biosciences) and data analyzed using Flowing software 2.5 [20].

\section{Measurement of reactive oxygen species (ROS)}

The changes in cellular ROS, in plants semi-purified fraction treated promastigotes have been compared with cells pre-incubated with NAC and without any treatment (control) using 2',7'dichlorodihydrofluorescein diacetate (H2DCFDA) (Sigma-Aldrich) $(20 \mu \mathrm{M})$ and analyzed by flow cytometer (BD FACSVerse, BD Biosciences) as described previously $[19,21]$ and analyzed by Flowing 2.5 version software [20]. The induction of ROS in promastigotes has been compared with positive inducer $\mathrm{H}_{2} \mathrm{O}_{2}$.

\section{Variation of the mitochondrial membrane potential $(\Delta \Psi m)$}

The variation of the accumulation of rhodamine 123 in promastigotes which is directly related to the mitochondrial membrane potential, was assessed in promastigotes after treatment with IC50 concentration of semi-purified fractions of A. mexicana $(50 \mu \mathrm{g} / \mathrm{ml})$, M. koenigii $(98 \mu \mathrm{g} / \mathrm{ml})$ and C. tamala $(200 \mu \mathrm{g} / \mathrm{ml})$ at different time points $(1 \mathrm{~h}, 3 \mathrm{~h}, 6 \mathrm{~h}$, and $12 \mathrm{~h})$ under standard conditions, as described previously [21]. Rhodamine 123 accumulation was monitored in a flow cytometer (BD FACSVerse, BD Biosciences, USA) and analyzed by Flowing 2.5 version software. The changes in mitochondrial $\Delta \Psi m$ in promastigotes have been compared in cells, preincubated with NAC and without any treatment (control).

\section{Detection of chromatin condensation}

The nuclear condensation in semi-purified fractions of all three plant treated promastigotes have been detected under FACS (BD FACSAria $^{T M}$, BD Biosciences) after staining with DAPI (4',6diamidino-2-phenylindole) by a slight modification of the methods described earlier [19] and cells were analyzed by Flowing 2.5 version software [20].

\section{Acridine orange/ethidium bromide staining method}

Promastigotes were seeded in 6 -well tissue culture plates $\left(1 \times 10^{6}\right.$ cells/well), treated without or with IC50 concentrations of the three semi-purified fractions individually for $24 \mathrm{~h}$, and the cells were washed with 1XPBS (Phosphate Buffered Saline). Then, the cells were stained with a mixture of acridine orange $(3 \mu \mathrm{g} / \mathrm{ml})$ and ethidium bromide $(10 \mu \mathrm{g} / \mathrm{ml})$. Place $10 \mu \mathrm{l}$ of cell suspension onto a microscopic slide, cover with a glass coverslip, and observed immediately in a fluorescence microscope (Carl Zeiss, Germany) using a fluorescein filter and a 40X objective. Higher or lower magnification could also be desired, counting on cell type. Each sample should be mixed just before microscopy and must be evaluated immediately [21].

\section{DNA fragmentation assay}

DNA fragmentation assay of L. donovani HePC-R promastigotes $\left(10^{7}\right.$ cells) without (control) or with IC50 concentrations of plants semipurified fraction were done by agarose gel electrophoresis to work out the fragments of DNA generated during apoptosis, total cellular DNA was isolated by a previously described method [22] and analyzed by agarose gel electrophoresis. Total DNA was mixed with tracking dye and loaded on $1 \%$ agarose gel containing ethidium bromide. The gel was run at $50 \mathrm{~V}$ for $2.5 \mathrm{~h}$.

Detection of the change in morphology by scanning electron microscopy (SEM)

L. donovani HePC-R promastigotes $\left(2 \times 10^{6}\right.$ cells $)$ in log phase were incubated at $22{ }^{\circ} \mathrm{C}$ in complete M199 medium, incubated with the 
three semi-purified fractions (IC50 against promastigotes) for $24 \mathrm{~h}$ and $48 \mathrm{~h}$ respectively. After incubation, promastigotes were centrifuged and the pellet was washed in 1 X cold PBS $(\times 2)$ and finally resuspended in $1 \mathrm{X}$ PBS. Promastigotes were then fixed with 2.5\% Gluteraldehyde (Sigma Aldrich), dehydrated in ethanol, critical point dried in CO2, mounted on stubs, sputtered with a thin platinum layer, and observed under a scanning electron microscope [19] (Model: ZEISS EVO-MA 10).

\section{RESULTS}

The semi-purified fractions of $A$. mexicana; M. koenigii and $C$. tamala inhibited the proliferation of $L$. donovani miltefosine resistant (HePC-R) promastigotes in vitro

Anti-proliferative effects of all three semi-purified fractions were evaluated on L. donovani HePC-R promastigotes, the causative agent of visceral leishmaniasis. It was found that semi-purified fractions of A. mexicana, M. koenigii, and C. tamala inhibited the proliferation of L. donovani HePC-R promastigotes in vitro. The $50 \%$ inhibitory concentration for A. mexicana, M. koenigii, and C. tamala against HePC-R promastigotes at $48 \mathrm{~h}$ were detected as $50 \pm 2.56 \mu \mathrm{g} / \mathrm{ml}$, $98 \pm 1.81 \mu \mathrm{g} / \mathrm{ml}$, and $200 \pm 1.57 \mu \mathrm{g} / \mathrm{ml}$, respectively (fig. $1 \mathrm{~A}$ ), which could restrict the proliferation of murine splenocytes only by $4.4 \pm 2.51 \%, 3.51 \pm 1 \%$ and $3.4 \pm 1.59 \%$ respectively at 96 h (fig. 1B). Our data indicated that the three compounds have noteworthy antiproliferative effects on L. donovani HePC-R promastigotes as well as non-toxic to normal murine primary cells. Data represent three independent experiments' mean \pm standard error. Statistical significance was determined by one-way ANOVA in $\mathrm{R}$ software followed by Tukey's test. Differences were considered to be statistically significant if the p-value is less than $0.05\left({ }^{*} \mathrm{p}<0.05\right.$ vs control).
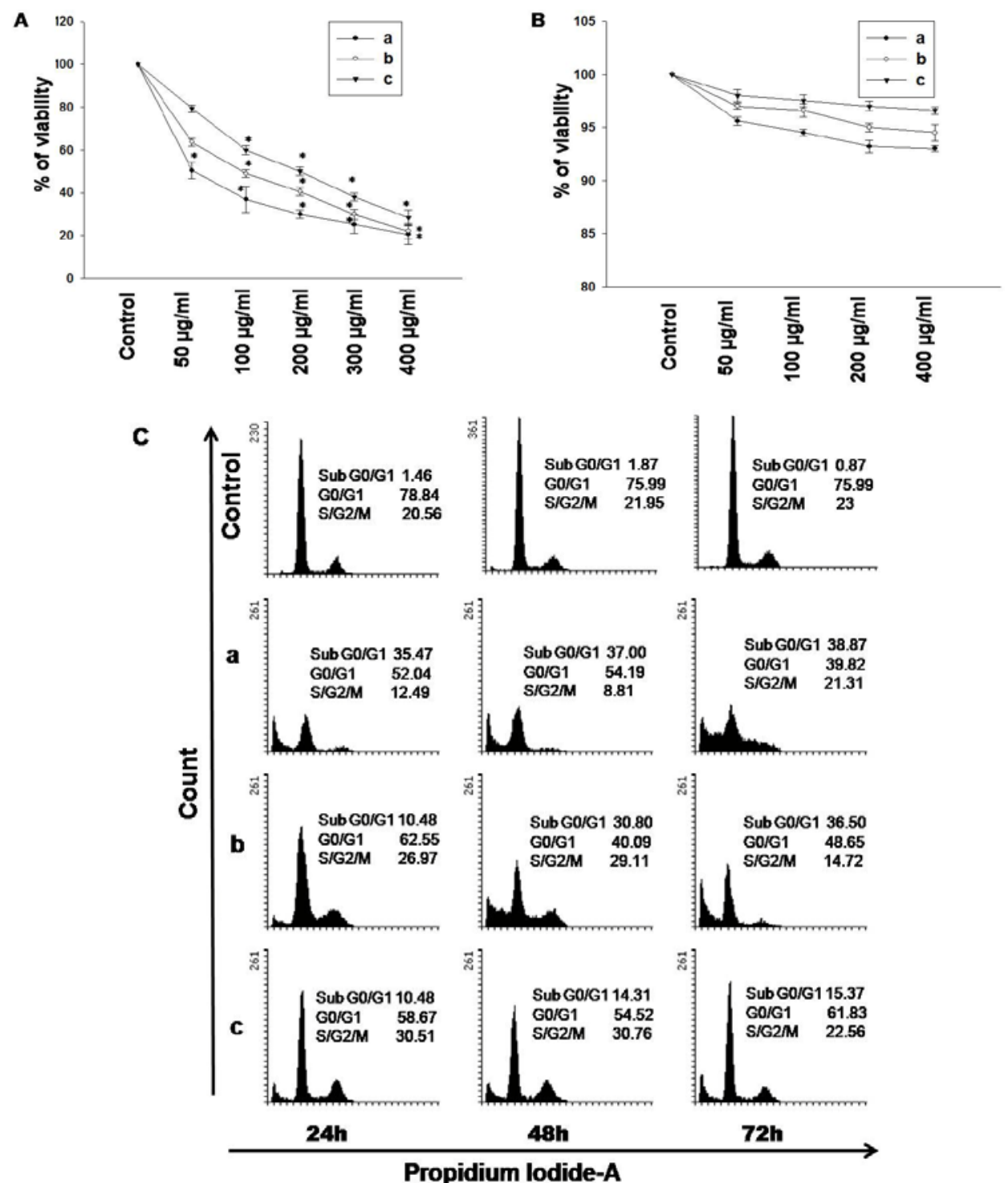

Fig. 1: In vitro anti-leishmanial activities of semi-purified fractions of (a) A. mexicana, (b) M. koenigii, and (c) C. tamala against L. donovani (HePC-R) promastigotes and toxicity assay towards murine splenocytes. (A) Dose-dependent inhibition and determination of $50 \%$ inhibitory concentration (IC50) by MTT assay. (B) Assessment of cytotoxicity of these three semi-purified fractions towards PHA induced splenocytes at IC50 concentrations $(* \mathbf{p}<0.05$ vs control). Data represent the mean \pm standard error of three independent experiments. Statistical significance was determined by one-way ANOVA in R software followed by Tukey's test. Differences were considered to be statistically significant if the $p$-value is less than $0.05(* p<0.05$ vs control). (C) The IC50 concentrations of these three semi-purified fractions disrupted the cell cycle progression in L. donovani (HePC-R) promastigotes $\left(10^{6}\right.$ cells/well) with an increase in sub G0/G1 phase population in comparison to the DMSO control $(0.2 \%, v / v)$; promastigotes were incubated for $24 \mathrm{~h}, 48 \mathrm{~h}$ and $72 \mathrm{~h}$ in complete M199 medium in the presence or absence of treatments 
The semi-purified fractions (IC50) of A. mexicana; M. koenigii and $C$. tamala induced alteration in cell cycle phases in HePC-R promastigotes

The IC50 concentration of semi-purified fractions of A. mexicana, M. koenigii, and $C$. tamala altered the cell cycle phases in promastigotes with a substantial increase in cell death time-dependently in comparison to DMSO control. Cell-cycle analysis complemented the cytotoxicity of the compounds obtained from MTT and 7AAD assay. It demonstrated that IC50 concentration of all three semi-purified fractions of A. mexicana, M. koenigii, and C. tamala $(50 \mu \mathrm{g} / \mathrm{ml} ; 98$ $\mu \mathrm{g} / \mathrm{ml}$ and $200 \mu \mathrm{g} / \mathrm{ml}$ respectively) caused promastigotes to remain as resting in G0/G1 phase and inhibited their entry into the $\mathrm{S}$ phase time-dependently at $24 \mathrm{~h}, 48 \mathrm{~h}$, and $72 \mathrm{~h}$ with a substantial increase in cell death, compared with DMSO treated control culture. At $24 \mathrm{~h}$ the test compounds started blockage the entry of promastigotes into the $S$ phase from G0/G1. Our results suggested that all three semipurified fractions preferentially disrupted the cell cycle phases in $L$. donovani HePC-R promastigotes followed by death in vitro (fig. 1C).

Semi-purified fractions of A. mexicana; M. koenigii and C. tamala exhibited substantial anti-promastigote activity

The viability of $L$. donovani HePC-R promastigotes was checked by staining with 7-AAD. $50 \mu \mathrm{g} / \mathrm{ml}$ concentrations of A. mexicana, $M$. koenigii, and $C$. tamala could increase the percentage of dead cells by $47.94 \pm 2.8 \%, 33.78 \pm 4.42 \%$, and $12.32 \pm 5.38 \%$, respectively (fig. $2 \mathrm{~A}$ ).

The semi-purified fractions (IC50) of A. mexicana; M. koenigii and $C$. tamala caused a decrease in total protein content in HePC-R promastigotes

The total cellular protein content of treated promastigotes was determined and compared with control promastigotes taking the unstained group as reference. It has been found that the treatment with semi-purified fractions could reduce the percentage of promastigotes with positively charged groups of proteins and were estimated as $11.71 \pm 5.04 \%$ (A. mexicana), $16.45 \pm 3.72 \%$ (M. koenigii), and $26.55 \pm 2.3 \%$ (C. tamala) in comparison to $99.02 \pm 0.462 \%$ in control culture at $48 \mathrm{~h}$. Moreover, semi-purified plant fractions also decreased the MFI in treated promastigotes. The MFI also dropped down to 65.68 (A. mexicana), 12.94 (M. koeniggi) and 26.54 (C. tamala) from 863.23 (control) in treated promastigotes at $48 \mathrm{~h}$ of treatment (fig. 2B).

The semi-purified fractions (IC50) of A. mexicana; M. koenigii and $C$. tamala caused the accumulation of lipids in HePC-R promastigotes

As semi-purified fractions of all three plants exhibited substantial anti promastigote activity in vitro, and reduced the total protein, we were interested to examine its effect on lipid metabolism in promastigotes. Nile red was used for studying lipid metabolism. At $48 \mathrm{~h}$ treated promastigotes were estimated with high neutral lipid content in comparison to the control promastigotes culture. The semi-purified fractions were also found to increase the MFI when treated and estimated for the neutral lipid droplets content (control vs treated) in promastigotes. The MFI was found to increase to 2109.69 (A. mexicana), 1640 (M. koeniggi) and 913.98 (C. tamala) from 52.80 in untreated (control) promastigotes at $48 \mathrm{~h}$ of treatment (fig. 2C).

The semi-purified fractions (IC50) of A. mexicana, M. koenigii, and $C$. tamala induced oxidative stress in L. donovani HePC-R promastigotes

The induction of ROS in HePC-R promastigotes was verified in semipurified fractions treatment-induced death, ROS was trapped inside cells using cell-permeant probe H2DCFDA with or without pretreatment of NAC the known antioxidants. Treatment of promastigotes with IC50 concentration of semi-purified fractions of A. mexicana, M. koenigii, and C. tamala revealed an elevation in ROS production time-dependently. The level of ROS in a semi-purified fraction of $A$. mexicana treated promastigotes increased to 1.62 -fold within $1 \mathrm{~h}$ of treatment and reached 1.78 -fold at $3 \mathrm{~h}, 3.06$-fold at $6 \mathrm{~h}$, and 2.83 -fold at $12 \mathrm{~h}$. In the semi-purified fraction of M. koenigii treated, promastigotes increased to 2.03 -fold within $1 \mathrm{~h}$ of treatment and reached 3.88-fold at $3 \mathrm{~h}, 1.72$-fold at $6 \mathrm{~h}$, and 1.64-fold in $12 \mathrm{~h}$. The semi-purified fraction of $C$. tamala treated promastigotes increased to 2.73-fold within $1 \mathrm{~h}$ of treatment and reached 3.16-fold at $3 \mathrm{~h}, 3.24$-fold at $6 \mathrm{~h}$, and 2.6-fold in $12 \mathrm{~h}$. Pretreatment of promastigotes with the antioxidant NAC before treatment with these semi-purified fractions, ROS generation was abrogated in comparison to treated cells (fig. 3A).
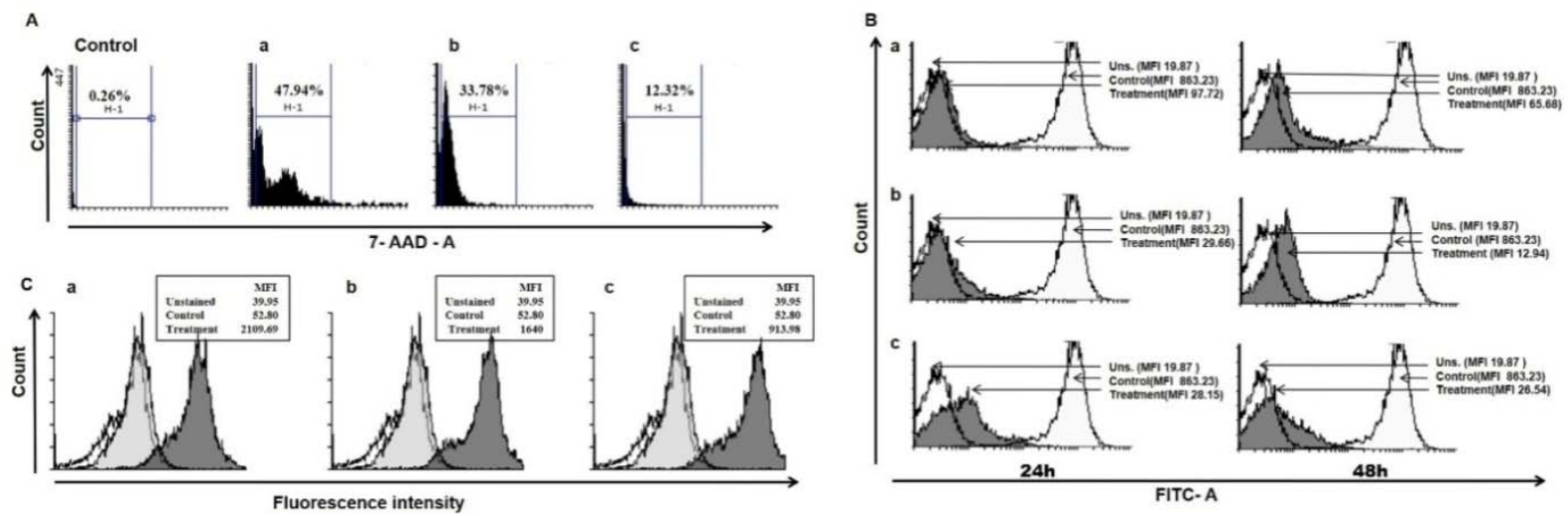

Fig. 2: Effect of semi-purified fractions of (a) A. mexicana, (b) M. koenigii, and (c) C. tamala on HePC-R promastigotes: (A) in vitro cytotoxicity assay: by using 7-AAD (7-amino actinomycin D). The lowest concentrations $(50 \mu \mathrm{g} / \mathrm{ml})$ of semi-purified fractions of (a) $A$. mexicana, (b) M. koenigii, and (c) C. tamala could increase the percentage of dead cells $47.94 \%, 33.78 \%$, and $12.32 \%$ respectively and

dead cells found in control culture only $0.26 \%$. (B) Biochemical parameters (Protein content): a decrease in total protein content (positively charged primary amines) was estimated in treated promastigotes in FACS by FITC. (C) Percentage of yellow fluorescence for neutral hydrophobic lipid domains were estimated in FACS by Nile red staining. All three semi-purified fractions significantly increased the percentage of promastigotes with high neutral lipid droplets containing promastigotes

The semi-purified fractions (IC50) of A. mexicana; M. koenigii and C. tamala induced ROS caused alteration in mitochondrial membrane potential

The study of mitochondrial membrane potential has become a focus of apoptosis regulation as many investigations demonstrated a major functional impact of mitochondrial alterations on apoptosis. Mitochondria are critical for the survival of any cell as they act as the warehouse of ATP. The proton gradient across the inner mitochondrial membrane is crucial during oxidative phosphorylation as the source for ATP production. Thus, the maintenance of mitochondrial membrane potential $(\Delta \Psi m)$ is 
essential for this chemical transformation as well as for cell survival [23]. The incorporation of rhodamine 123 has been used to determine the changes in mitochondrial membrane potential. All the three semi-purified fractions of $A$. mexicana, $M$. koenigii, and $C$. tamala caused loss of mitochondrial membrane potential and were found to be continued from $1 \mathrm{~h}$ to $12 \mathrm{~h}$ of treatment. The alteration of $\Delta \Psi \mathrm{m}$ in the semi-purified fraction of $A$. mexicana treated promastigotes was found to be started gradually from $1 \mathrm{~h}$ (2.31-fold decrease in comparison to control promastigotes) continued to the extent of decrease by 2.4 -fold after $3 \mathrm{~h}, 3.83$-fold after $6 \mathrm{~h}$ and even 5.2 -fold loss after $12 \mathrm{~h}$ of treatment. The alteration of $\Delta \Psi m$ in the semi-purified fraction of $M$. koenigii treated promastigotes decreased by 2.44-fold after 1 h, 3.44-fold after 3 h, 3.76-fold after 6 $\mathrm{h}$, and even 5.32 -fold loss after $12 \mathrm{~h}$ of treatment in comparison to control promastigotes. The alteration of $\Delta \Psi m$ in the semi-purified fraction of $C$. tamala treated promastigotes was also found started from $1 \mathrm{~h}$ (1.2-fold decrease in comparison to control promastigotes) continued to the extent of decrease by 1.94-fold after $3 \mathrm{~h}, 2.53$-fold after $6 \mathrm{~h}$ and even 3.79-fold loss after $12 \mathrm{~h}$ of treatment. Interestingly, pre-incubation with NAC prevented the alteration of plant semi-purified fractions-induced mitochondrial membrane potential, which could be further linked with the prospect of ROSmediated inhibition of $L$. donovani promastigotes by the semipurified fractions (fig. 3B).
A
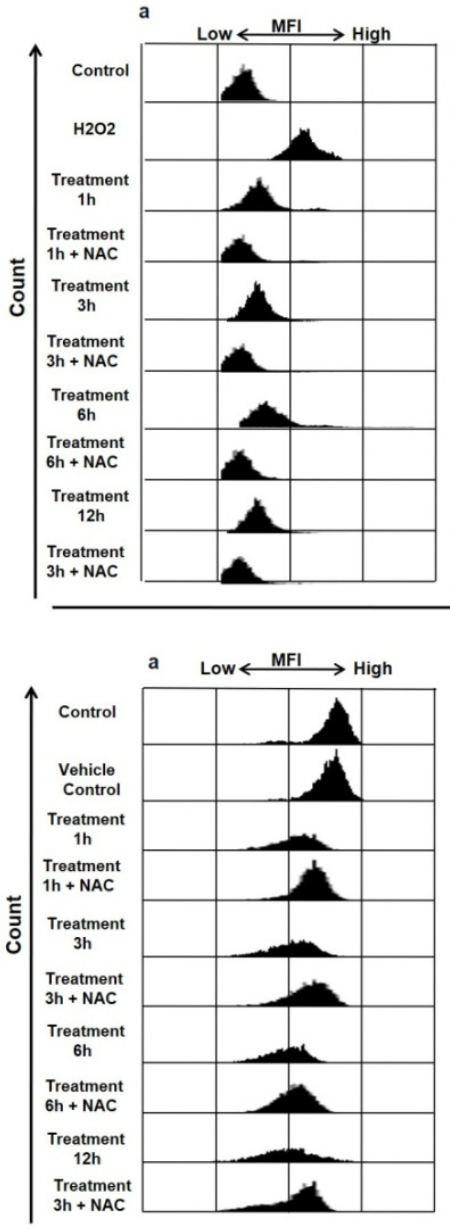

b
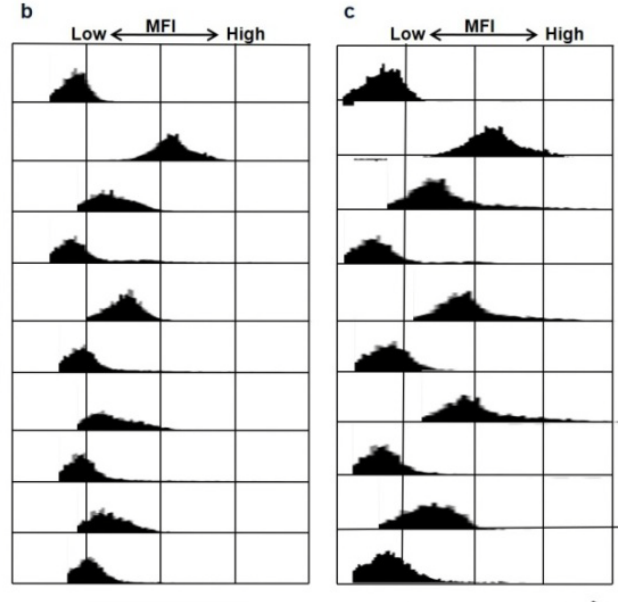

FITC-A

b Low $\stackrel{\text { MFI }}{\longleftrightarrow}$ High

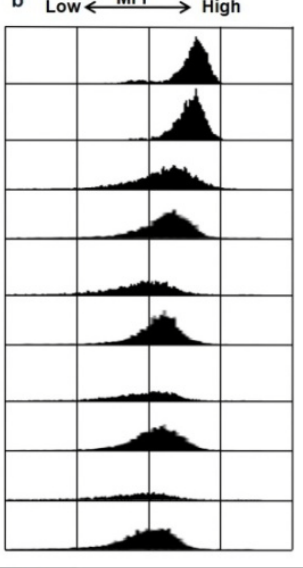

c Low $\stackrel{\mathrm{MFI}}{\longrightarrow}$ High

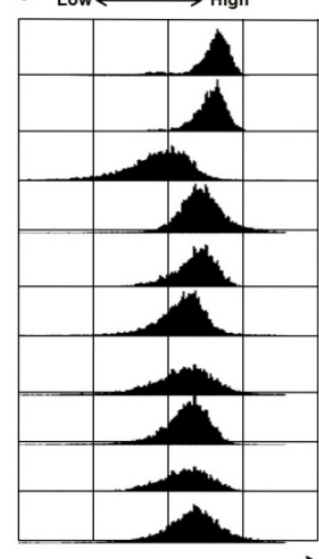

FITC-A

Fig. 3: The IC50 concentrations of semi-purified fractions of (a) A. mexicana, (b) M. koenigii, and (c) C. tamala (A) induced ROS production in Leishmania donovani HePC-R promastigotes $\left(10^{6}\right.$ cells/well): $1,3,6$, and $12 \mathrm{~h}$ treatment of promastigotes revealed an elevation of intracellular reactive oxygen species (ROS) time-dependently. However, pretreatment of promastigotes with the antioxidant NAC before treatment with these three semi-purified fractions individually, ROS generation was found to be abrogated in each time point. Positive

control was obtained by the addition of $\mathrm{H}_{2} \mathrm{O}_{2}(30 \mathrm{~min})$. (B) Loss of $\Delta \Psi \mathrm{m}$ in L. donovani (HePC-R) promastigotes $\left(10^{6} \mathrm{cells} / \mathrm{well}\right)$ : The variation in the accumulation of rhodamine 123 in promastigotes was quantified by a flow cytometer. These three semi-purified fractions caused depolarization of mitochondrial membrane potential within $1 \mathrm{~h}$ of treatment individually. Interestingly, preincubation with NAC prevented the alteration of semi-purified fractions-induced mitochondrial membrane potential

The semi-purified fractions (IC50) of A. mexicana; M. koenigii and C. tamala caused DNA condensation in HePc-R promastigotes

Staining with DAPI revealed the appearance of chromatin condensation at a much higher rate in treated promastigotes than in the control. During apoptosis, the cleavage patterns of genomic DNA are typical of internucleosomal DNA digestion by endonucleases and are considered as a characteristic of apoptosis that's preceded by chromatin condensation. The semi-purified fractions of $A$. mexicana, M. koenigii, and C. tamala treated promastigotes showed DAPI positive condensed nucleus at $48 \mathrm{~h}$ (fig. 4A).
The semi-purified fractions (IC50) of A. mexicana; M. koenigii and C. tamala caused apoptosis of Leishmania Promastigotes, observed in acridine orange/ethidium bromide staining

The treated promastigotes were stained with acridine orange/ethidium bromide to verify the apoptosis in treated promastigotes. After the treatment with IC50 concentrations of semi-purified fractions of A. mexicana, M. koenigii, and C. tamala individually for $24 \mathrm{~h}$, the majority of cells exhibited diffused orangecolored promastigotes or orange fluorescence, while in control promastigotes, green fluorescence was observed. Treated 
promastigotes developed orange and orange-red fluorescence, indicating membrane disruption (fig. 4B). These results support that all three semi-purified fractions could induce apoptosis at IC50 concentrations.

The semi-purified fractions (IC50) of A. mexicana; M. koenigii and $C$. tamala induced morphological alterations in $L$. donovani HePC-R promastigotes

Scanning electron microscopy exposed specific morphological alterations in promastigotes after treatment with the IC50 concentration of semi-purified fraction of A. mexicana, M. koenigii, and C. tamala plants for $24 \mathrm{~h}$ and $48 \mathrm{~h}$. Promastigotes appeared irregularly shaped with gradual loss of cell membrane and flagella concerning the control cultured flagellated and slender promastigotes (fig. 4C).

The semi-purified fractions (IC50) of A. mexicana; M. koenigii and $C$. tamala treatment resulted in the fragmentation of DNA

Tests for an additional hallmark of apoptosis is the internucleosomal genomic DNA degradation, shown by the experimental group which had nucleosome-sized DNA fragments, giving a DNA ladder-like pattern identified by agarose gel electrophoresis of DNA from the cells treated with IC50 concentration of semi-purified fractions of $A$. mexicana, M. koenigii and C. tamala plants (fig. 4D).
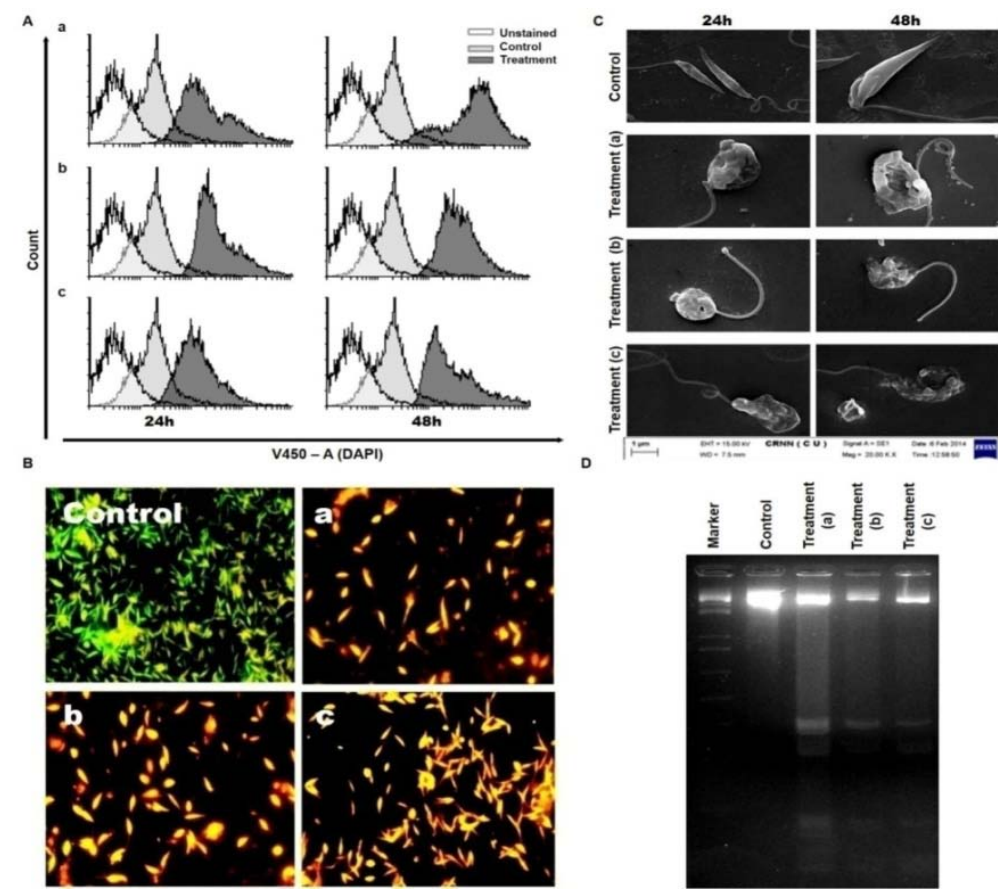

Fig. 4: The IC50 concentrations of semi-purified fractions of (a) A. mexicana (b) M. koenigii and (c) C. tamala against Leishmania donovani (HePc-R) promastigotes: (A) induced DNA condensation: DNA condensation was found increased time-dependently at $24 \mathrm{~h}$ and $48 \mathrm{~h}$ of treatment detected by DAPI staining in FACS (B) Acridine orange/Ethidium Bromide-stained cells were observed under a fluorescence microscope (40X). Viable cells (no treatment) show green fluorescence (control). Apoptotic cells show orange and yellow fluorescence (treated). Cells were observed under a fluorescence microscope (40X). (C) Substantial morphological alterations observed by scanning electron microscopy (SEM): Time-dependent dramatic and drastic changes in the promastigote shape, plasma membrane topography, and loss of flagella were evidenced after treatment in comparison to control promastigote; $(0.2 \%$ (v/v) DMSO was used for control experiments. Magnification: $2000 \mathrm{~K} \mathrm{X}$, scale bars: $1 \mu \mathrm{M}$ (images are representative of three independent experiments). (D) Detection of

DNA fragmentation: Ladder-like DNA fragmentation profile detected in agarose gel from untreated and treated promastigotes

\section{DISCUSSION}

Increasing issues of drug resistance to the limited anti-leishmanial drugs imply the search for novel leishmanicidal agents. Earlier, there are multiple reports of antileishmanial and antiprotozoal activities of various plants extracts [7-10]; but no previous reports of $A$. mexicana, M. koenigii, $C$. tamala extracts against drug-resistant $L$. donovani parasites. The present study was intended to identify the anti-leishmanial effect of three traditionally used medicinal plants used in folklore by the tribal healer or common villagers of the Malda district in India. In vitro leishmanicidal activities against miltefosine resistant (HePC-R) promastigotes were done by the semi-purified fractions of the plants; Argemone mexicana, Murraya koenigii, and Cinnamomum tamala. The semi-purified fractions of $A$. mexicana; $M$. koenigii and $C$. tamala exhibited substantial antileishmanial activity with an estimated IC50 of $50 \pm 2.56 \mu \mathrm{g} / \mathrm{ml}$, $98 \pm 1.81 \mu \mathrm{g} / \mathrm{ml}$, and $200 \pm 1.57 \mu \mathrm{g} / \mathrm{ml}$, respectively as compared to the standard drugs amphotericin B $0.29 \pm 0.05 \mu \mathrm{g} / \mathrm{ml}$ and pentamidine $5.09 \pm 0.09 \mu \mathrm{g} / \mathrm{ml}$ [9] (fig. $1 \mathrm{~A})\left({ }^{*} \mathrm{p}<0.05 \mathrm{vs}\right.$ control) with no cytotoxic effect on murine splenocytes (fig. 1B). The minimal concentration that is $50 \mu \mathrm{g} / \mathrm{ml}$ of these three semi-purified fractions could increase the percentage of dead cells by $47.94 \pm 2.8 \%$, $33.78 \pm 4.42 \%$, and $12.32 \pm 5.38 \%$ respectively of $L$. donovani (HePCR) promastigotes at $48 \mathrm{~h}$ of treatment as estimated by flowcytometric approach, staining with 7AAD (fig. 2A). The IC50 concentrations of these three semi-purified fractions caused a decrease in total protein content (fig. 2B) and accumulation of lipids in cells as analyzed by FACS, staining with Nile red (fig. 2C). Accumulation of lipids in cells could be related to the alterations of plasma membrane biophysical properties and also the degradation of abnormal lipids. Thus, the accumulation of lipid in cells as the consequence of the treatment with these three semi-purified fractions could hamper the plasma membrane integrity, resulting in cell death. This result discriminates live promastigotes from dead one on a membrane integrity basis. Reactive oxygen species (ROS) have been reported as the key modulator against the Leishmania parasites [24]. Interestingly, the IC50 concentration of these three semi-purified fractions against the promastigotes was found to induce oxidative stress by producing the highest amount of (ROS) measured in FACS, (fig. 3B) and this result was also found correlated with the alteration in mitochondrial membrane potential in treated promastigotes (fig. 3B). Treatment with IC50 of these three semi- 
purified fractions was found to alter the cell cycle phases caused promastigotes to remain resting in G0/G1 cells and inhibited their entry into the $S$ phase time-dependently (fig. $1 \mathrm{C}$ ) which was a clear indication of apoptosis in promastigotes which was also supported by acridine orange/EtBr staining experiment (fig. 4B). Fragmentation of the nucleus is considered the hallmark of apoptosis [23, 25]. IC50 of these three semi-purified fractions caused DNA condensation, observed by DAPI staining (fig. 4A) and DNA fragmentation giving the DNA a ladder-like pattern identified by agarose gel electrophoresis (fig. 4D) and also the morphological alterations were observed by SEM in $L$. donovani HePc-R promastigotes (fig. 4C). This data substantially indicated that these three semi-purified fractions were proficient in arresting the proliferation of $L$. donovani promastigotes by inducing apoptosis (fig. 4). These three active semi-purified fractions against promastigotes were also found non-toxic to normal murine splenocytes (fig. 1B). However, investigation on bioactive molecules from Argemone mexicana, Murraya koenigii, and Cinnamomum tamala has not been done extensively. So, further phytochemical and pharmacological investigations are necessary for the search of active anti-leishmanial constituents from Argemone mexicana, Murraya koenigii, and Cinnamomum tamala. The consequences of this study can be used as a reference for further phytochemical and pharmacological investigations within the effort for the search of novel anti-leishmanial leads.

\section{CONCLUSION}

The results of the present study established that plant materials are potential sources of novel and selective agents which contribute a lot to primary health care and most likely are promising agents for the treatment of leishmaniasis. Semi-purified fractions of A. mexicana, $M$. koenigii, and C. tamala showed potential activities against drugresistant (Miltefosine) Leishmania species and also no toxicity on splenocytes authorized its specific anti-promastigote effect which is induced by reactive oxygen species (ROS). The study will help to develop potent, non-toxic plant-derived anti-leishmanial drugs. Further investigations are needed for the identification and isolation of active plant constituents.

\section{ACKNOWLEDGEMENT}

I like to acknowledge Prof. Narayan Ghorai, WBSU for sharing his expertise on natural product research. We are also thankful to the Director, Centre for Research in Nanoscience and Nanotechnology (CRNN), the University of Calcutta for access to the scanning electron microscopy facility. I am very thankful to UGC/MANF, Govt. of India for providing me the fellowship [Ref: MANFMUS-WES-5322/2011-12].

\section{FUNDING}

Nil

\section{AUTHOR CONTRIBUTION}

The author confirms sole responsibility for the following: study conception and design, data collection, experimentation, analysis and interpretation of results, manuscript preparation, critical revision of the article, and final approval of the version to be published.

\section{CONFLICT OF INTERESTS}

The authors declare no conflict of interest in the publication of this manuscript.

\section{REFERENCES}

1. World Health Organization. World Health Report. Geneva: WHO; 2012. p. 186-91.

2. Lira R, Sundar S, Makharia A, Kenney R, Gam A, Saraiva E, et al. Evidence that the high incidence of treatment failures in Indian kala-azar is due to the emergence of antimony-resistant strains of Leishmania donovani. J Infect Dis 1999;180:564-7.

3. Rojas R, Valderrama L, Valderrama M, Varona MX, Ouellette M, Saravia NG. Resistance to antimony and treatment failure in human Leishmania (Vienna) infection. J Infect Dis 2006;193:1375-83.
4. Hadighi R, Mohebali M, Boucher P, Hajjaran H, Khamesipour A, Ouellette M. Unresponsiveness to glucantime treatment in Iranian cutaneous leishmaniasis due to drug-resistant Leishmania tropica parasites. PLoS Med 2006;3:659-67.

5. Cox PA, Balick MJ. The ethnobotanical approach to drug discovery. Sci Am 1994;270:82-7.

6. Kavishankar GB, Lakshmidevi N, Murthy SM, Prakash HS, Niranjana SR. Diabetes and medicinal plants-a review. J Pharm Biomed Sci 2011;3:65-80.

7. Tahir AE, Ibrahim AM, Satti GMH, Theander TG, Kharazmi A Khalid SA. The potential antileishmanial activity of some Sudanese medicinal plants. Phytother Res 1998;12:576-9.

8. Weniger B, Robledo S, Arango GJ, Deharo E, Aragon R, Munoz V, et al. Antiprotozoal activities of colombian plants. J Ethnopharmacol 2001;78:193-200.

9. Sharma KR. Phytotoxic, antioxidant, antibacterial, and antileishmanial activities of Euphorbia hirta from Chitwan district Nepal. Asian J Pharm Clin Res 2020;13:146-9.

10. Chan Bacab MJ, Pena Rodriguez LM. Plant natural products with leishmanicidal activity. Nat Prod Rep 2001;18:674-88.

11. Rajvaidhya S, Nagori BP, Singh GK, Dubey BK, Desai P, Jain S. A review on Argemone mexicana Linn. an Indian medicinal plant. Int J Pharm Sci Res 2012;3:2494-501.

12. Nadkarni KM. Indian Materia Medica. Popular Prakashan, Mumbai; 1976. p. 196.

13. Gopalan S, Kulanthai K, Sathasivam G. A review on phytochemical constituents of $M$. koenigii roots: rutaceae. Int J Curr Pharm Rev Res 2015;6:41-8.

14. Roy S, BOSE S, Sarkar D, Mandal S, Sarkar S, Mandal SK Formulation and evaluation of anti-acne gel containing Murraya koeinigii extract. Int J Curr Pharm Res 2020;12:108-13.

15. Soni R, Mehta NM, Srivastava DN. Effect of ethanolic extract of Cinnamomum tamala leaves on wound healing in STZ induced diabetes in rats. Asian J Pharm Clin Res 2013;6:39-42.

16. Panchawat S, Sisodia SS. In vitro antioxidant activity of Saraca asoca Roxb. De wilde stem bark extracts from various extraction processes. Asian J Pharm Clin Res 2010;3:231-3.

17. Raajshree KR, Durairaj B. Evaluation of the antityrosinase and antioxidant potential of zinc oxide nanoparticles synthesized from the brown seaweed-Turbinaria conoides. Int J Appl Pharm 2017;9:116-20.

18. Seifert K, Perez Victoria FJ, Stettler M, Sanchez Canete MP, Castanys S, Gamarro F, et al. Inactivation of the miltefosine transporter, LdMT, causes miltefosine resistance that is conferred to the amastigote stage of Leishmania donovani and persists in vivo. Int J Antimicrob Agents 2007;30:229-35.

19. Sultana SS, Ghosh J, Chakraborty S, Mukherjee D, Dey S, Mallick $\mathrm{S}$, et al. Selective in vitro inhibition of Leishmania donovani by a semi-purified fraction of wild mushroom Grifola frondosa. Exp Parasitol 2018;192:73-84.

20. Perttu Terho, Centre for Biotechnology, Turku, Finland. Available from: www.flowingsoftware.com [Last accessed on 05 May 2021].

21. Mirunalini S, Karthishwaran K, Vaithiyanathan V. Antiproliferative potential of Pergularia daemia (Forsk.) on human oral epidermoid carcinoma (KB) cells by inducing apoptosis and modifying oxidant-antioxidant status. Asian J Pharm Clin Res 2014;7:89-95.

22. Rotureau B, Gego A, Carme B. Trypanosomatid protozoa: a simplified DNA isolation procedure. Exp Parasitol 2005;111:207-9.

23. Sen N, Das BB, Ganguly A, Mukherjee T, Tripathi G, Bandyopadhyay S, et al. Camptothecin induced mitochondrial dysfunction leading to programmed cell death in unicellular hemoflagellate Leishmania donovani. Cell Death Differ 2004;11:924-36.

24. Sagbo IJ, Mbeng WO. Chemical constituents, antioxidative, cytotoxic, and genotoxic effects of Miscanthus capensis roots extract. Asian J Pharm Clin Res 2019;12:227-32.

25. Shaha C. Apoptosis in Leishmania species and its relevance to disease pathogenesis. Indian J Med Res 2006;123:233-44. 